\title{
GAIA Level 3a Assessment of Gestational Age
}

National Cancer Institute

\section{Source}

National Cancer Institute. GAIA Level 3a Assessment of Gestational Age. NCI Thesaurus. Code C128016.

GAIA Level 3a Assessment of Gestational Age is defined by the presence at least one of the following criteria: a) Certain last menstrual period (LMP) date with third trimester scan (28 0/7 weeks or more); OR b) Certain LMP date with confirmatory second trimester fundal height (FH); OR c) Certain LMP date with birth weight; OR d) Uncertain LMP date with first trimester physical examination. 